\title{
A RIQUEZA DAS FORMIGAS RELACIONADA AOS PERÍODOS SAZONAIS EM CAXIUANÃ DURANTE OS ANOS DE 2006 E 2007.
}

\author{
SÉRGIO RODRIGO QUADROS DOS SANTOS ${ }^{1}$, MARIA ISABEL VITORINO ${ }^{2}$, ANA YOSHI \\ HARADA $^{3}$, ADRIANO MARLISOM LEÃO DE SOUZA ${ }^{4}$ E EVERALDO BARREIROS DE SOUZA $^{2}$.
}

${ }^{1}$ Universidade Federal de Campina Grande (UFCG), Campina Grande, PB, Brasil, ${ }^{2}$ Universidade Federal do Pará (UFPA), Belém, PA, Brasil,

${ }^{3}$ Museu Paraense Emilio Goeldi (MPEG), Belém, PA, Brasil, ${ }^{4}$ Universidade Federal Rural da Amazônia (UFRA), Belém, PA, Brasil,

sergiosanntos@yahoo.com.br,vitorino@ufpa.br,ahara@museu-goeldi.br,marlisoms@yahoo.com.br,everaldo@ufpa.br.

Recebido Abril de 2011 - Aceito Março de 2012

\begin{abstract}
RESUMO
A variabilidade temporal da fauna de formigas coletadas em Caxiuanã-PA, durante o protocolo de formigas de serrapilheira do Projeto TEAM/Caxiuanã, foram estudadas a partir das condições meteorológicas locais observadas nos meses de janeiro a abril (estação chuvosa), e julho a outubro (estação menos chuvosa), para os anos de 2006 e 2007. Para isso, foram utilizados dados meteorológicos da torre micrometeorológica de Caxiuanã. Durante a estação chuvosa, notou-se o predomínio de elevados valores de precipitação e umidade do solo, e baixas temperaturas do ar. Na estação menos chuvosa, observou-se comportamento oposto ao período chuvoso. Em geral, observou-se que a frequência de formigas é maior quando há redução da precipitação e da umidade do solo; e do aumento da temperatura do ar. Os gêneros das formigas Crematogaster, Hypoponera, Pheidole e Solenopsis apresentaram maior quantidade de indivíduos. Percebeu-se ainda, que as correlações estatísticas com função polinomial de segunda ordem, entre as variáveis atmosféricas e a frequência de formigas, mostram claramente que estas ocorrem de modo inverso com a precipitação e a umidade do solo, e direta com a temperatura do ar. Assim, os resultados deste estudo corroboram a alta variação da abundância dos quatro gêneros de formigas supracitados, em função das variáveis atmosféricas em áreas tropicais.

Palavras-Chave: Formigas, Atmosfera, Amazônia, Precipitação
\end{abstract}

\begin{abstract}
THE ANTS WEALTH IN RELATION TO SEASONAL CAXIUANÃ PERIODS DURING YEARS 2006 AND 2007

The temporal variability of ant fauna collected in Caxiuanã-PA during the leaf litter ants protocol of the Project TEAM/Caxiuanã were studied based on the local meteorological conditions observed during the months from January to April (rainfall season), and July to October (dry season) to 2006 to 2007. Data from the micrometeorological tower at Caxiuanã were used. During the rainfall season the predominance of high values of precipitation and soil moisture and low values of air temperatures is noted. In the dry season the opposite behavior compared to the rainfall season is observed. In general, it was observed that the frequency of ants is greater when precipitation and soil moisture are reduced and air temperature is increased. Ants of the genera Crematogaster, Hypoponera, Pheidole and Solenopsis have higher number of individuals. It was noted also that the statistical correlations using second-order polynomial function, between atmospheric variables and the frequency of ants, occurred in reverse mode for precipitation and soil moisture, and direct for the air temperature. Our results corroboreted to the high abundance of the four upper mentioned ant genera in relation to the environmental atmospheric condictions in tropical regions.
\end{abstract}

Keywords: Ants, Atmosphere, Amazon, Precipitation 


\section{INTRODUÇÃO}

A floresta Amazônica é uma importante região do globo por sua conhecida fonte de energia para a circulação geral da atmosfera, desempenhando um papel ativo em relação às trocas energéticas dentro do sistema solo-planta-atmosfera (Molion, 1985; Sá et al., 1986). A bacia amazônica contém uma variedade de ecossistemas e grande riqueza em termos de diversidade biológica e étnica. Esta região contém a mais extensa floresta tropical do globo e possui recursos hídricos abundantes, com mais de cinco milhões de quilômetros quadrados, e responde por aproximadamente um quarto das espécies animais e vegetais do planeta (Marengo, 2006).

Apresenta dominância de clima quente e úmido, onde os gradientes horizontais de temperaturas do ar são muito pequenos, há muita nebulosidade e bastante precipitação convectiva. A umidade do ar, embora com valores altos, sofre variações acentuadas no tempo e no espaço. Estas condições atmosféricas são moduladas por sistemas meteorológicos que ocorrem desde a escala local, meso até a grande escala, tais como: os cumulunimbus isolados, as linhas de instabilidade, a Zona de Convergência Intertropical, entre outros (Cohen et al., 1995; Mota e Souza, 1996; Machado et al., 2002).

As formigas (família Formicidae) fazem parte da ordem Hymenoptera, a qual constitui um dos grupos mais diversos e bem adaptados entre os insetos (Borror e De Long, 1988). Dentre a extraordinária diversidade de insetos nas regiões tropicais, as formigas se destacam apresentando grande riqueza em espécies e uma variedade de funções ecológicas nos ecossistemas (Hölldobler e Wilson, 1990). Podem ser detritívoras, predadoras e se alimentarem de produtos vegetais (néctar e sementes), ou cultivarem fungos (tribo Attini). Entre as cultivadoras de fungos tem-se algumas espécies que podem causar severas desfolhas às plantas (Schultz e McGlynn, 2000).

O estudo de Souza et al. (2007) registrou 36 espécies de formigas pertencentes aos gêneros Crematogaster, Gnamptogenys e Pachycondyla para a Região de Caxiuanã-Pa. Segundo Majer (1997), as formigas são indicadores ambientais, pois apresentam sensibilidade às mudanças no meio ambiente, além de possuírem relação com a estrutura das comunidades de outros organismos.

Considerando a alta riqueza das formigas, principalmente nas áreas tropicais, há necessidade de inventários e outros estudos para se conhecer o que existe e o melhor entendimento de suas funções ecológicas nos ecossistemas. Neste contexto, o objetivo deste trabalho visa verificar como as variáveis meteorológicas em Caxiuanã-Pa, influenciam na frequência de indivíduos de formigas.

\section{DADOS E METODOLOGIA}

A área em estudo está localizada na Floresta Nacional de

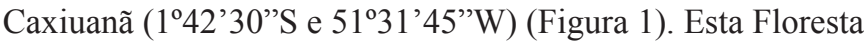

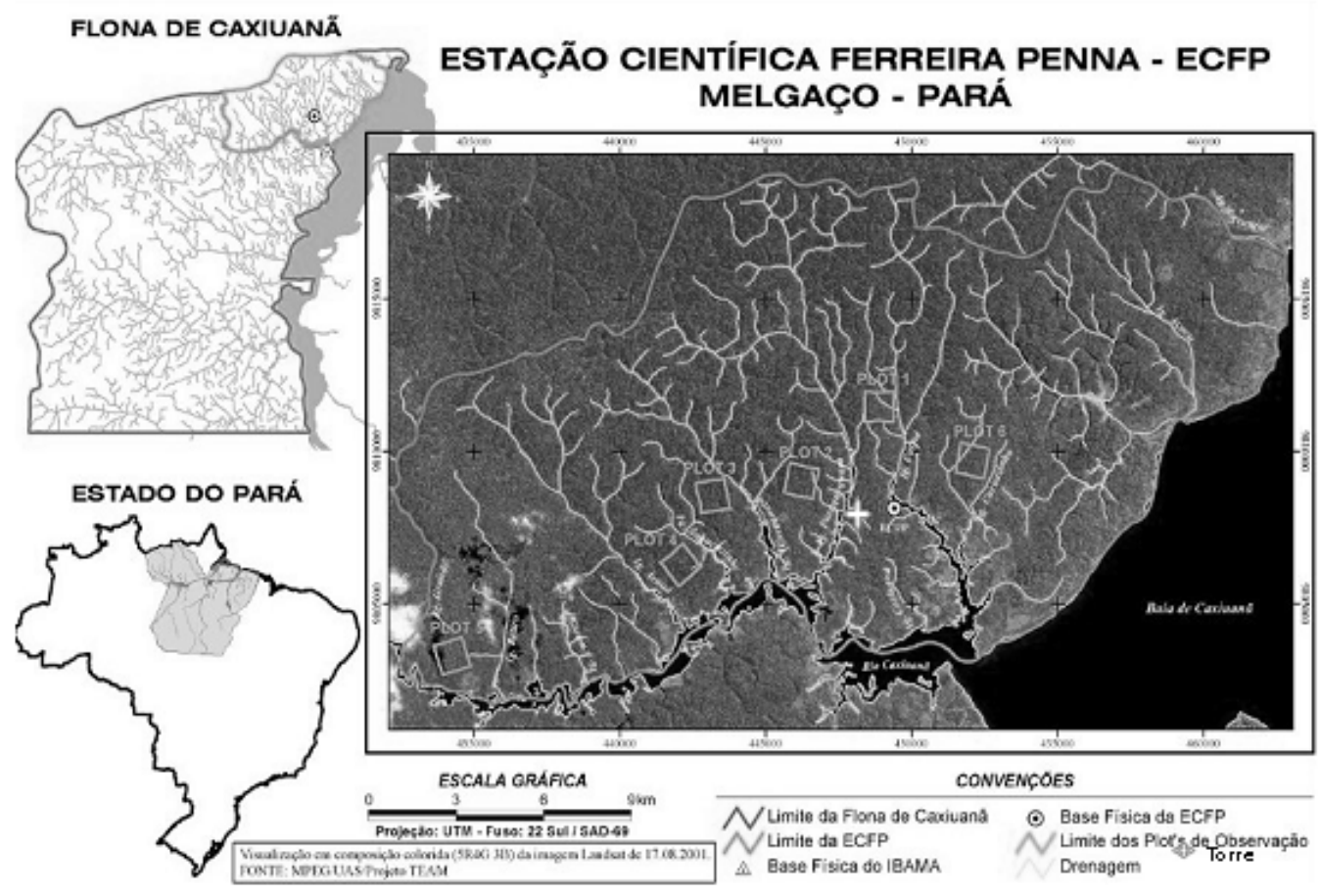

Figura 1- Mapa de localização da ECFPn e das áreas de coleta de formigas. 
é constituída, em $85 \%$ de sua área, por floresta densa de terra firme, com dossel médio das árvores entre 30 e $40 \mathrm{~m}$ de altura, as quais, entretanto só interceptam pouco mais de $10 \%$ da precipitação incidente (Moraes et al., 1997). Segundo Ruivo et al. (2000), na região predominam solos classificados como Latossolos, que variam de bem a moderadamente drenados, cor bruno-amarelo-escuro a vermelho-amarelado; a textura varia de arenosa à argilosa. Esta Região apresenta duas estações bem distintas ao longo do ano, uma chuvosa compreendida nos meses de dezembro a maio, e outra menos chuvosa que se estende de junho a novembro (Costa et al., 2006).

Para análise das condições meteorológicas locais foram utilizados totais mensais de precipitação pluviométrica $(\mathrm{mm})$, médias da temperatura do ar $\left({ }^{\circ} \mathrm{C}\right)$ e umidade do solo $\left(\mathrm{m}^{3} \mathrm{~m}^{-3}\right)$ na profundidade de $5 \mathrm{~cm}$, para os meses representativos ao período chuvoso (janeiro e abril) e menos chuvoso (julho e outubro), nos anos de 2006 e 2007.

As aquisições destes dados foram feitas através dos seguintes sensores, CS616-L50 que mede a umidade do solo, HMP45AC, que mede a temperatura do ar e o pluviógrafo TB4-L RAIN GAUGE, que mede a precipitação. Estes sensores são de baixa freqüência e estão conectados a um datalogger modelo CR10X (Campbell Scientific). Os mesmos fazem parte de uma estação meteorológica automatizada que está instalada em uma torre micrometeorológica de $54 \mathrm{~m}$ de altura. Os dados foram coletados em campanhas mensais e armazenados a cada 30 minutos, em seguida foram tratados e encaminhados a um banco de dados.

A torre micrometeorológica e todos os seus sensores pertencem ao Programa LBA (Large Scale BiosphereAtmosphere in Amazon Program) (Von Randow et al., 2002 ), o qual envolve pesquisas interdisciplinares sobre o funcionamento da Amazônia como entidade regional, com respeito aos ciclos da água, carbono, gases do efeito estufa e nutrientes; e como as alterações dos usos da terra e do clima afetam o funcionamento biológico, físico e químico dos ecossistemas amazônicos.

As formigas foram coletadas no decorrer do desenvolvimento do protocolo de formigas de serrapilheira do Projeto TEAM/Caxiuanã (Projeto de Ecologia, Avaliação e Monitoramento de Florestas Tropicais), em seis parcelas de
$1 \mathrm{~km}^{2}$ em torno de 20 a $50 \mathrm{~km}$ da torre micrometeorológica (Souza et al., 2007). Neste estudo foi considerada a frequência de ocorrência de indivíduos para as seis parcelas, visando melhor conhecer a relação entre as formigas e as variáveis meteorológicas mencionadas anteriormente.

O método de amostragem utilizado neste estudo foi o extrator de Winkler, por ser apropriado para usar em ambientes de floresta, na coleta de formigas que utilizem a serrapilheira para forragear ou para abrigar-se (Bestelmeyer et al., 2000), e foi considerado o mais eficiente em número de espécies coletadas (Delabie et al., 2000; Fisher et al., 2000). Foram realizadas quatro coletas anuais (janeiro, abril, julho e outubro) de $2006 \mathrm{e}$ 2007. Em cada área (plote) foram instalados quatro transectos de $100 \mathrm{~m}$, onde foram estabelecidos 10 pontos, distanciados $10 \mathrm{~m}$ entre si para a coleta das amostras. Em cada ponto de amostragem foi retirado $1 \mathrm{~m}^{2}$ de serrapilheira, a qual foi penerada durante 2 minutos. O material penerado foi transferido para um saco de tecido e transportado ao laboratório da Estação Científica Ferreira Penna (ECFPn), onde foi colocado em um saco telado e exposto no extractor de mini-Winkler durante $48 \mathrm{~h}$. Após este período, o material coletado foi armazenado em frasco vedável e conduzido ao Laboratório de Sistemática e Ecologia de Formigas do MPEG, onde foi triado e identificado. Todo material foi depositado na Coleção de Invertebrados do MPEG.

Testes estatísticos de regressão linear, com funções logarítmicas, exponenciais, lineares, potenciais e polinomiais de segunda ordem foram aplicados para a determinação da equação que melhor represente a relação entre as formigas e as variáveis meteorológicas na flora de Caxiuanã (Tabela 1). Porém, foram usadas apenas as regressões polinomiais de segunda ordem, por apresentar resultados mais significantes de $\mathbf{r}^{2}$.

\section{RESULTADOS E DISCUSSÕES}

O total pluviométrico em Caxiuanã (Figura 2) para 2006 e 2007, durante os meses correspondentes a estação chuvosa e menos chuvosa foi de $638 \mathrm{~mm}$ e $121 \mathrm{~mm}$, e de $365 \mathrm{~mm}$ e 159 $\mathrm{mm}$, respectivamente. Observou-se ainda que para 2006 e 2007 , a temperatura do ar média na estação chuvosa e menos chuvosa foi de $22,4^{\circ} \mathrm{C}$ e $24,4^{\circ} \mathrm{C}$, e de $23,7^{\circ} \mathrm{C}$ e $24,2^{\circ} \mathrm{C}$, respectivamente.

Tabela 1 - Valores de $\mathrm{r}^{2}$ para as variáveis meteorológicas e as Formicidae.

\begin{tabular}{cccc}
\hline Funções & $\begin{array}{c}\text { Precipitação } x \\
\text { Formicidae }\end{array}$ & $\begin{array}{c}\text { Temperatura do } \\
\text { ar x Formicidae }\end{array}$ & $\begin{array}{c}\text { Umidade do solo } \\
\text { x Formicidae }\end{array}$ \\
\hline Exponencial & 0,10 & 0,30 & 0,66 \\
\hline Linear & 0,13 & 0,42 & 0,70 \\
\hline Polinomial & 0,68 & 0,67 & 0,72 \\
\hline Logarítmica & 0,35 & 0,41 & 0,70 \\
\hline Potencial & 0,28 & 0,29 & 0,65 \\
\hline
\end{tabular}




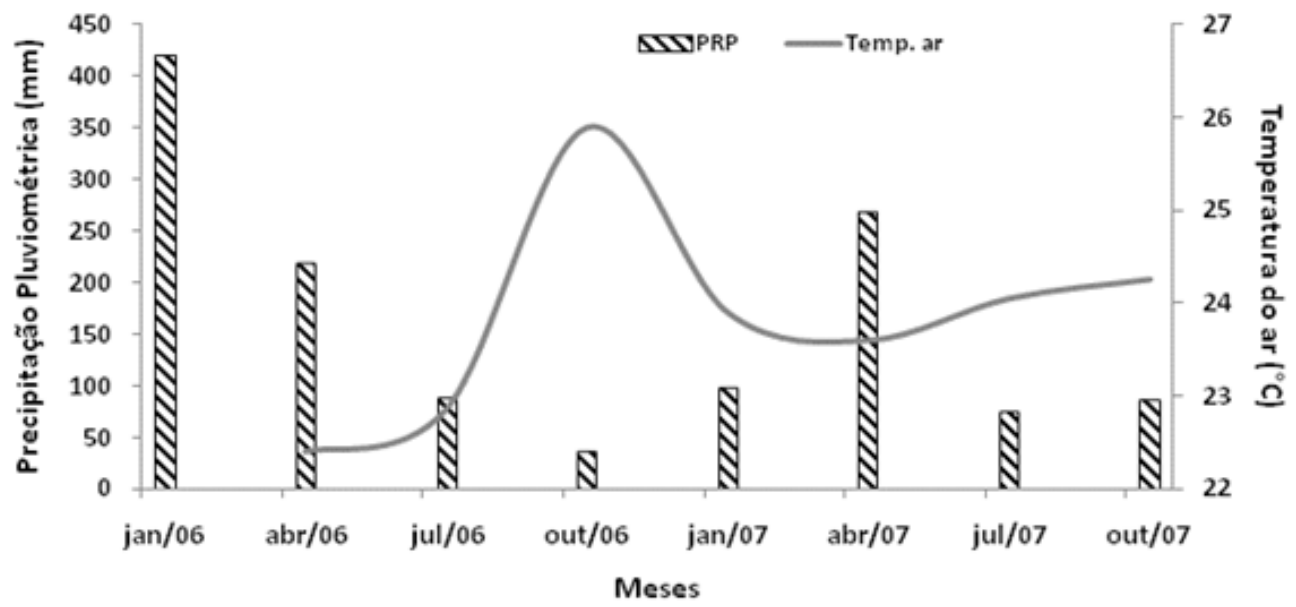

Figura 2 - Variação temporal da precipitação pluviométrica e da temperatura do ar, durante os meses de janeiro, abril, julho e outubro de 2006, e 2007 respectivamente.

Nota-se que os totais pluviométricos da estação chuvosa de 2006 foram maiores que o do ano de 2007, porém quando são analizados os totais da estação menos chuvosa verifica-se que foram menores em 2006 e maiores em 2007. Esta variabilidade sazonal da precipitação pode estar relacionada com a ocorrência do fenômeno ENOS (El Niño Oscilação Sul) e/ou com a atuação da OMJ (Oscilações de Madden e Julian), fenômenos cuja suas consequências para a Amazônia foram estudadas por Souza e Ambrizzi (2006).

Verificou-se ainda que a média da temperatura do ar durante a estação chuvosa de 2006 foi menor que a de 2007, isto pode estar relacionado com o aumento de nebulosidade que ocorre na estação chuvosa, reduzindo a quantidade de radiação solar na superfície terrestre, consequentemente foram observadas temperaturas do ar mais baixas do que na estação menos chuvosa. Segundo Nobre et al. (1991), a variabilidade temporal da precipitação, associada a ocorrência de eventos extremos (excesso ou escassez) prolongados, fazem parte do controle natural dos ecossistemas. Por isso, ter compreensão da variabilidade climática dos extremos de chuvas e secas pode fornecer elementos para a preservação e desenvolvimento sustentável de uma região, de modo a lidar com os episódios adversos.

A umidade do solo é um elemento importante, pois regula as atividades dos organismos e limita a distribuição dos mesmos dentro de um ecossistema (Odum, 1988). Neste sentido, nota-se na Figura 3 que durante a estação chuvosa e menos chuvosa de Caxiuanã os valores da umidade do solo, em 2006 e 2007 , foram de $0,446 \mathrm{~m}^{3} \cdot \mathrm{m}^{-3}$ e $0,422 \mathrm{~m}^{3} \cdot \mathrm{m}^{-3}$ na estação chuvosa, e $0,360 \mathrm{~m}^{3} \cdot \mathrm{m}^{-3}$ e $0,376 \mathrm{~m}^{3} \cdot \mathrm{m}^{-3}$ na menos chuvosa, respectivamente.

Notou-se que na estação chuvosa, de ambos os anos, a umidade do solo foi maior do que na estação menos chuvosa, pois na estação chuvosa há maior frequência do molhamento do dossel e desta forma há maior disponibilidade hídrica para a floresta. Vale ressaltar que, os valores de umidade do solo foram maiores na estação chuvosa de 2006 , pois os totais pluviométricos dessa estação apresentaram maiores valores que o de 2007. Uma análise análoga pode ser feita para a estação menos chuvosa.

A Figura 4 mostra que a frequência de formigas é maior na estação chuvosa de 2007 e menor na de 2006. Porém a estação menos chuvosa de 2006 apresenta uma maior frequência de formigas do que a de 2007. Verificou-se, anteriormente, que a estação chuvosa de 2007 e a estação menos chuvosa de 2006 apresentaram menores valores de precipitação (Figura 2), de maneira análoga, essa análise pode ser feita para as demais variáveis meteorológicas em estudo.

Desta forma, a frequência de formiga é maior quando há menores valores da precipitação e umidade do solo, e maiores valores da temperatura do ar. Com a diminuição significativa da precipitação, a umidade do solo diminui principalmente nas camadas superiores do solo, havendo também um aumento da temperatura do ar e do solo. Assim, as formigas da floresta de Caxiuanã, mostram-se adaptadas ao clima quente e úmido da região, pois quanto mais quente e úmido, mesmo na estação menos chuvosa, a umidade do ar elevada que se origina da evapotranspiração favorece o desenvolvimento das Formicidae, fato que corrobora outros estudos realizados em áreas tropicais (Andersen, 1997). Portanto, os meses de julho e outubro de 2006 apresentaram maior frequência e riqueza de gêneros das formigas.

O estudo de Araújo et al. (2002) mostrou que a floresta Amazônica apresenta altas taxas de evapotranspiração, mesmo na estação menos chuvosa. Isto ocorre porque as árvores da Floresta Amazônica, na sua maioria, têm raízes profundas, atingindo o lençol freático nas camadas inferiores do solo. 


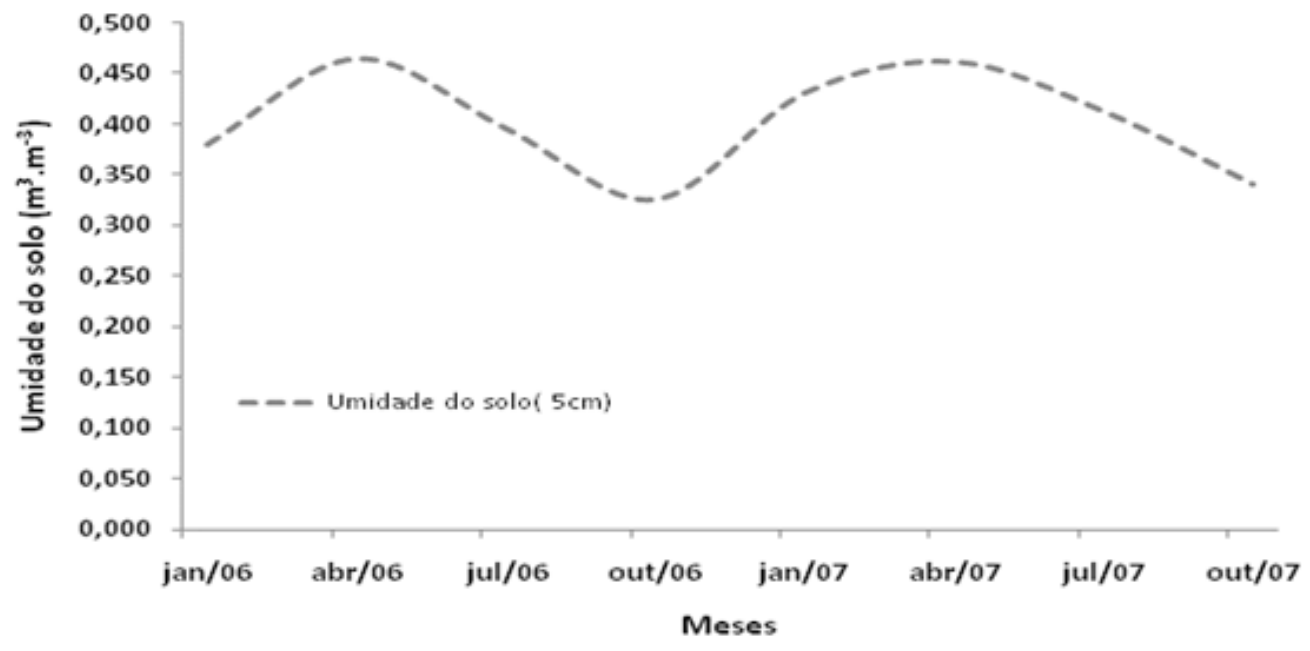

Figura 3 - Variação temporal da umidade do solo, para os meses de janeiro, abril, julho e outubro de 2006 e 2007.

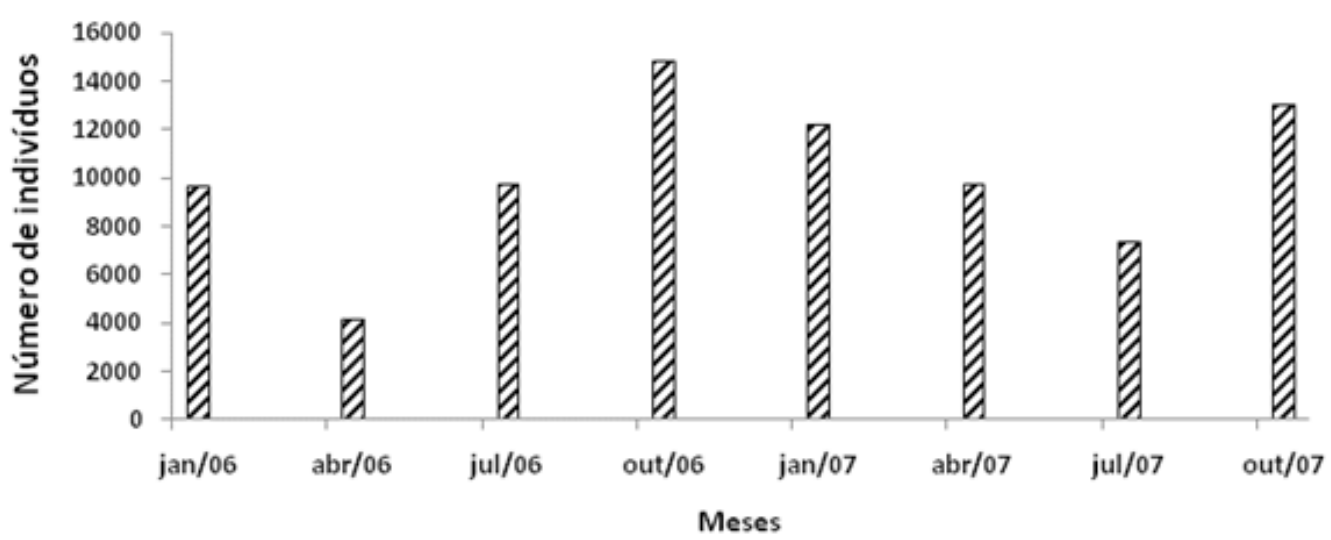

Figura 4 - Variação temporal das frequência de formigas na Flona de Caxiuanã -PA, durante 2006 e 2007.

Os gêneros mais abundantes em ambos os anos estudados foram os Crematogaster, Hypoponera, Pheidole e Solenopsis. Segundo Fernández (2003) estes gêneros possuem grande abundância, riqueza e capacidade adaptativa nos nichos ecológicos na Região neotropical, uma vez que as espécies desses gêneros apresentam grande diversidade de hábitos e habitats.

No ano de 2006 foram registrados na estação chuvosa $18 \%$ (Crematogaster), $8 \%$ (Hypoponera), 20\% (Pheidole) e 20\% (Solenopsis), e na estação menos chuvosa 22\% (Crematogaster), $8 \%$ (Hypoponera), $18 \%$ (Pheidole) e 20\% (Solenopsis). Em 2007, registrou-se 10\% (Crematogaster), 6\% (Hypoponera), 18\% (Pheidole) e 19\% (Solenopsis) na estação chuvosa, e 18\% (Crematogaster), 8\% (Hypoponera), 18\% (Pheidole) e 20\% (Solenopsis) na estação menos chuvosa (Tabela 2).

Fagundes et al. (2009) ressalta que, várias explicações podem ser dadas para a existência das variações temporais de espécimes de formigas no decorrer das estações. A disponibilidade de alimentação é um dos principais fatores relacionados à abundância das formigas. Comportamentos específicos, como hábitos nômades de armazenamento de alimentos ou especificidade de habitat podem determinar a permanência de uma espécie, durante e após a variação das condições atmosféricas locais.

A correlação polinomial de segunda ordem mostra que as Formicidae apresentam uma alta correlação com a precipitação (68\%), com a temperatura do ar (67\%) e com a umidade do solo (72\%) (Figura 5). Mostra-se claramente que estas ocorrem de modo inverso para a precipitação e para a umidade do solo, ou seja, à medida que um aumenta o outro diminui. Para a temperatura do ar foi observado uma relação diretamente proporcional, ou seja, quando esta variável atmosférica aumenta, a frequência da formiga também aumenta.

Desta forma, as condições atmosféricas em Caxiuanã influenciam a fauna de formigas, onde as mesmas respondem dinamicamente aos estímulos ou restrições impostas por estas 
Tabela 2 - Variação temporal dos gêneros mais abundantes de formigas nas estações chuvosa e menos chuvosa dos anos de 2006 e 2007.

\begin{tabular}{|c|c|c|c|c|}
\hline & \multicolumn{2}{|l|}{2006} & \multicolumn{2}{|r|}{2007} \\
\hline Gêneros & $\begin{array}{l}\text { Estação } \\
\text { Chuvosa }\end{array}$ & $\begin{array}{c}\text { Estação Menos } \\
\text { Chuvosa }\end{array}$ & $\begin{array}{l}\text { Estação } \\
\text { Chuvosa }\end{array}$ & $\begin{array}{c}\text { Estação Menos } \\
\text { Chuvosa }\end{array}$ \\
\hline Crematogaster & $18 \%$ & $22 \%$ & $10 \%$ & $18 \%$ \\
\hline Hypoponera, & $8 \%$ & $8 \%$ & $6 \%$ & $8 \%$ \\
\hline Pheidole & $20 \%$ & $18 \%$ & $18 \%$ & $18 \%$ \\
\hline Solenopsis & $20 \%$ & $20 \%$ & $19 \%$ & $20 \%$ \\
\hline Outros & $34 \%$ & $32 \%$ & $47 \%$ & $36 \%$ \\
\hline
\end{tabular}
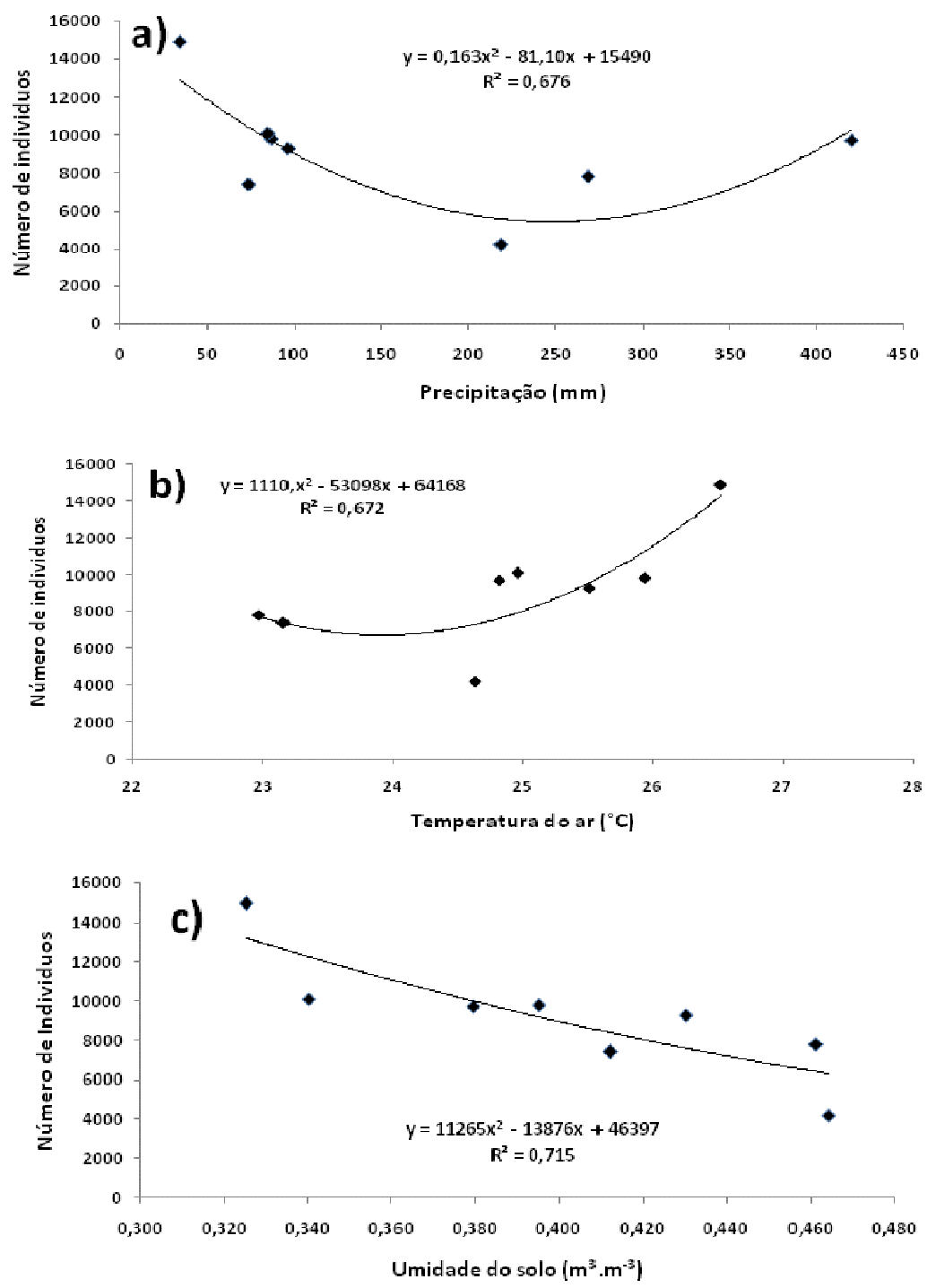

Figura 5 - Correlações estatísticas entre os indivíduos de formigas e as variáveis meteorológicas para os anos de 2006 a 2007. (a) Precipitação versus formiga; (b) temperatura do ar versus formiga; (c) umidade do solo versus formiga. 
condições atuantes. Dessa forma, os dados utilizados neste estudo corroboram a hipótese de Hölldobler e Wilson (1990) que sugerem que a temperatura e a umidade do ar e do solo criam um envelope de restrições para as diferentes espécies de formigas, influenciando na sua reprodução, distribuição e sobrevivência.

\section{CONCLUSÕES}

Este trabalho constatou que a sazonalidade atmosférica da precipitação, temperatura do ar e umidade do solo influenciaram na abundância de Formicidae na Floresta Nacional de Caxiuanã, no período de 2006 a 2007. Em geral, observou-se que a frequência de formigas é maior quando há redução da precipitação, da umidade do solo, e do aumento das temperaturas do ar. Os gêneros das formigas Crematogaster, Hypoponera, Pheidole e Solenopsis apresentaram maior quantidade de indivíduos em todos os períodos do ano.

As correlações estatísticas - função polinomial de segunda ordem entre as variáveis atmosféricas e a frequência de formigas mostram claramente que estas ocorrem de modo inverso a precipitação e a umidade do solo, e direto a temperatura do ar mostrando a ação das variáveis meteorológicas sobre a comunidade de formigas. Todavia, estudos de monitoramento mais prolongados são necessários para estabelecer um padrão da dinâmica biológica neste complexo ambiente amazônico.

\section{AGRADECIMENTOS}

O primeiro autor agradece ao Museu Paraense Emílio Goeldi e ao CNPq pela bolsa de pesquisa concedida através do Projeto Cenários. Os autores agradecem ao Museu Paraense Emílio Goeldi, IBAMA e ao projeto TEAM que proporcionaram todas as facilidades para o bom andamento da coleta de dados, ao Escritório Central do LBA, ao LIM-CPTECINPE e a faculdade de Meteorologia da UFPA pelo apoio às atividades experimentais em Caxiuanã.

\section{REFERÊNCIAS BIBLIOGRÁFICAS}

ANDERSEN, A. N. Using ants as bioindicators: Multiscale issues in ant community ecology. Conservation Ecology [on line], 1(1): 8. 1997.

ARAUJO, A. C.; OBRE, A. D.; KRUIJT, B.; ELBERS, J. A.; DALLAROSA, R.; STEFANI, P.; RANDOW, C.; MANZI, A.O.; CULF, A.D.; GASH, J.H.C.; VALENTINI, R.; KABAT, P. Comparative measurements of carbon dioxide fluxes from two nearby towers in a central Amazonian rainforest: The Manaus LBA site. Journal of Geophysical Research, v. 107, p. 5820. 2002.
BESTELMEYER, B. T.; AGOSTI, D.; LEEANNE, F.; ALONSO, T.; BRANDÃO, C. R. F.; BROWN, W. L.; DELABIE, J. H. C.; SILVESTRE, R. Field techniques for the study of ground-living ants: An Overview, description, and evaluation. In: AGOSTI, D.; MAJER, J.D.; TENNANT, A.; SCHULTZ, T.R. (Eds). Ants: Standard methods for measuring and monitoring biodiversity. Washington, DC., USA: Smithsonian Institution Press. p. 122-144. 2000.

BORROR, D. J.; DE LONG, D. M. Introdução ao estudo dos insetos. Chicago . Edgard Blücher LTDA, 653 p. 1988.

COHEN, J. C. P.; SILVA DIAS, M. A. F.; NOBRE, C. A. Environmental conditions associated with Amazonian squall lines: a case study. Monthly Weather Review. n.123, p.3163-3174. 1995.

COSTA,A.C.L;BRAGA, A.P; GONÇALVES, P.H.L; COSTA, R. F; SILVIA JUNIOR, J. A; MALHI, Y. S; ARAGÃO, L. E. O. E; MEIR, P. Estudos Hidrometeorológicos em uma floresta tropical chuvosa na Amazônia - Projeto ESECAFLOR. Revista Brasileira de Meteorologia, v. 21, n.3b. p. $283-290.2006$.

DELABIE, J. H. C.; AGOSTI, D.; NASCIMENTO, I. C. Litter ant communities of the Brazilian Atlantic rain forest region. In: AGOSTI, D.; MAJER, J. D.; ALONSO, L.; SCHULTZ, T. (Eds.), Sampling ground-dwelling ants: case studies from the world's rain forests. Bulletin. 2000.

FAGUNDES, R.; SANTOS, N. B. E. ; SILVA, G. L.; MAIA, A. C. R.; SANTOS, J. F. L; RIBEIRO, S. P. Efeito das mudanças climáticas sazonais no forrageio de Formigas em uma área de mata estacional semidecidual Montana. In: CONGRESSO DE ECOLOGIA DO BRASIL, 9., 2009, São Lourenço - MG. Anais... São Lourenço - MG: SBEO, 1 CD-ROM. 2009.

FERNANDEZF. (ed.). Introduccion a las Hormigas de la region Neotropical. Bogota: Instituto de Investigación de Recursos Biologicos Alexander von Humboldt, xxvi; 398p. 2003. FISHER, B. L.; MALSCH, A. K. F.; GADAGKAR, R.; DELABIE, J.H.C.; VASCONCELOS, H.L.; MAJER, J.D. Applying The All Protocol. In: AGOSTI, D. ; MAJER, J. D. ; TENNANT, A.; SCHULTZ, E T. R. (Eds). Ants: standard methods for measuring and monitoring biodiversity. Smithsonian Institution Press, Washington, DC., USA. p. 207-214. 2000.

HÖLLDOBLER, B.; WILSON. E. O. The ants. Cambridge: The Belknap Press of Havard University Press, p. 731. 1990.

MACHADO, L. A. T.; LAURENT, H.; LIMA, A. A. Diurnal March of the Convection Observed During TRMMWETAMC/ LBA. Journal of Geophysical Research, v. 107, n. D20, 0.1029/2001JD000338. 2002.

MAJER, J. D. The use of pitfall traps for sampling ants - a critique. Memoirs of the Museum of Victory, v. 56, n. 2, p.323-329. 1997. 
MARENGO, J. A. Mudanças climáticas globais e seus efeitos sobre a biodiversidade: caracterização do clima atual e definição das alterações climáticas para o território brasileiro ao longo do século XXI - IBAMA, Brasília: MMA, 2006.

MOLION, L. C. B. Micrometeorology of an Amazon rain forest. In: DICKINSON, R. E. (ed). The geophysiology of Amazonia: vegetation and climate interactions. Chichester, UK: John Wiley; Sons, p. 255-269. 1985.

MORAES, J. C.; COSTA, J. P. R.; ROCHA, E. J. P.; SILVA, I. M. O. Estudos Hidrometeorologicos na Bacia do Rio Caxiuanã. Em: LISBOA, P. L. B. CAXIUANÃ. (Ed.) Belém: Publicação do Museu Paraense Emilio Goeldi, MCT/ CNPq, p 85-95. 1997.

MOTA, M. A. S.; SOUZA, P. F. S. Influência da precipitação nas características termodinâmicas da atmosfera durante um mês seco. In: CONGRESSO BRASILEIRO DE MEteorologiA, 9., Campos do Jordão-SP, Anais... SBMET, p. 1136-1138. 1996.

NOBRE, C. A.; SELLERS, P. J.; SHUKLA, J. Amazonian deforestation and regional climate change. Journal of Climate, v. 4, p.957-987. 1991.

ODUM, E. P. Ecologia, 1. ed. Rio de Janeiro, RJ. Guanabara, 434p. 1988.

RUIVO, M. L. P.; QUANZ, B.; SALES, M. E. C; MEIR, P. Solos dos sítios do experimento ESECAFLOR. In: LISBOA, P. L. B. (org.). Caixuanã: populações tradicionais, meio físico $\&$ diversidade biológica. Belém: Museu Paraense Emilio Goeldi, p.207-213. 2000.
SÁ, L. D. A.; MANZI, A. O.; VISWANADAM, Y. Partição de fluxos de calor sensível e de calor latente acima da floresta amazônica de terra firme. São José dos Campos, INPE, julho (INPE-3972-PRE/970). 1986.

SCHULTZ, T. R.; MCGLYNN, T. P. The interactions of ants with other organisms. In: AGOSTI, D.; MAJER, J. D.; ALONSO, L. E. ; SCHULTZ, T. R. eds. Ants: standard methods for measuring and monitoring biodiversity. Washington, Smithsonian Institution. p.35-44. 2000.

SOUZA, E. B.; AMBRIZZI, T. Modulation of the intraseasonal raifall over tropical Brasil by the Madden-Julian Oscillation. Iternational Journal of Climatology, v.26, p1759-1776. 2006.

SOUZA, J. L. P. ; MOURA, C. A. R. ; HARADA, A. ; FRANKLIN, E. Diversidade de espécies dos gêneros de Crematogaster, Gnamptogenys e Pachycondyla (Hymenoptera: Formicidae) e complementaridade dos métodos de coleta durante a estação seca numa estação ecológica no Estado do Pará, Brasil. Acta Amazonica, v. 37, n.4, p. 649-656. 2007.

VON RANDOW, C. ; SÁ, L. D. A. ; PRASAD, G. S. S. D. ; MANZI, A. O. ; ARLINO AND B. KRUIJT, P. R. A. Scale variability of atmospheric surface layer fluxes of energy and carbon over a Tropical Rain Forest in Southwest Amazonia: diurnal conditions, Journal of Geophysical Research, v.107, n. D20, p. 29.1-29.12, 4 September. 2002. 